\title{
Significance of gluon density for soft and hard processes at LHC
}

\section{G. I. Lykasov*†}

Joint Institute for Nuclear Research, Dubna, Moscow region, 141980, Russia

E-mail: lykasovejinr.ru

\section{A. A. Grinyuk}

Joint Institute for Nuclear Research, Dubna, Moscow region, 141980, Russia

E-mail: andrei.grinyuk@gmail.com

\section{A. V. Lipatov}

Skobeltsyn Institute of Nuclear Physics, Lomonosov Moscow State University, 119991 Moscow,

Russia

Joint Institute for Nuclear Research

E-mail: artem/lipatov@mail.ru

\section{N. P. Zotov}

Skobeltsyn Institute of Nuclear Physics, Lomonosov Moscow State University, 119991 Moscow, Russia

E-mail:zotov@theory.sinp.msu.ru

We study the role of the non-perturbative input to the transverse momentum dependent (TMD) gluon density in hard processes at the LHC. Special attention is paid to phenomenological applications of the obtained TMD gluon density to some LHC processes, which are sensitive to the gluon content of a proton.

24th International Workshop on Deep-Inealstic Scattering and Related Subjects. 11-15 April 2016, DESY, Hamburg, Germany

* Speaker.

${ }^{\dagger}$ A footnote may follow. 


\section{- Introduction}

In the present paper we continue our studies[1,2] and investigate the role of the non-perturbative input to the TMD gluon density in description of hard processes at the LHC. We improve the initial TMD gluon distribution proposed earlier to describe LHC data on the inclusive charged hadron spectra at higher transverse momenta $2.5<p_{T}<4.5 \mathrm{GeV}$ and numerically extend it to the whole kinematical region using the CCFM gluon evolution equation. The CCFM equation is the most suitable tool for our study since it smoothly interpolates between the small- $x$ Balitsky-Fadin-KuraevLipatov[5] (BFKL) gluon dynamics and the conventional Dokshitzer-Gribov-Lipatov-AltarelliParisi[6] (DGLAP) one. We extract additional parameters from a fit to the LHC data on the inclusive $b$-jet production taken by the CMS and ATLAS Collaborations at high $p_{T}$ and $\sqrt{s}=7 \mathrm{TeV}$. We supply the obtained TMD gluon density with the corresponding TMD valence and sea quark distributions calculated in the approximation, where the sea quarks occur in the last gluon splitting. Finally, we discuss several phenomenological applications of the proposed TMD parton densities to hard LHC processes that are most sensitive to the quark and gluon content of the proton.

- Starting non-perturbative TMD gluon density The proposed early [1] non-perturbative gluon density is not able to describe the LHC data on the inclusive spectrum of charged hadrons at higher transverse momenta $2.5<p_{T}<4.5 \mathrm{GeV}$ even if additional pertubative QCD (pQCD) corrections [7, 8] are taken into account at $p_{T}>2 \mathrm{GeV}$. Moreover, gluon density (1) much faster decreases when $\mathbf{k}_{T}^{2}$ grows compared to the solution of the BFKL equation outside of the saturation region [5, 6, 9]. Therefore, we modify the gluon density given by (1) at $\left|\mathbf{k}_{T}\right|>2-3 \mathrm{GeV}$ to describe the LHC data on the charged hadron production at $2.5<p_{T}<4.5 \mathrm{GeV}$. Then we match it with the TMD gluon obtained in[9] as the solution of the linear BFKL equation at low $x$, which results in flatter $\mathbf{k}_{T}^{2}$ behavior. The modified starting TMD gluon density can be presented in the following form:

$$
\begin{gathered}
f_{g}^{(0)}\left(x, \mathbf{k}_{T}^{2}, \mu_{0}^{2}\right)=c_{0} c_{1}(1-x)^{b} \times \\
\times\left[R_{0}^{2}(x) \mathbf{k}_{T}^{2}+c_{2}\left(R_{0}^{2}(x) \mathbf{k}_{T}^{2}\right)^{a / 2}\right] \exp \left(-R_{0}^{2}(x) \mathbf{k}_{T}^{2}-d\left[R_{0}^{2}(x) \mathbf{k}_{T}^{2}\right]^{3 / 2}\right)+ \\
+c_{0}\left(\frac{x}{x_{0}}\right)^{n} \exp \left[-k_{0}^{2} \frac{R_{0}(x)}{\left|\mathbf{k}_{T}\right|}\right] f_{g}\left(x, \mathbf{k}_{T}^{2}\right)
\end{gathered}
$$

where $k_{0}=1 \mathrm{GeV}, \mu_{0}^{2}=1.1 \mathrm{GeV}^{2}$, and $n \simeq 0.81$. The function $f_{g}\left(x, \mathbf{k}_{T}^{2}\right)$ obeying the BFKL equation at not very large $\mathbf{k}_{T}^{2}$ reads[9]

$$
f_{g}\left(x, \mathbf{k}_{T}^{2}\right)=\alpha_{s}^{2} x^{-\Delta} t^{-1 / 2} \frac{1}{v} \exp \left[-\frac{\pi \ln ^{2} v}{t}\right]
$$

where $t=14 \alpha_{s} N_{c} \zeta(3) \ln (1 / x), v=\left|\mathbf{k}_{T}\right| / \Lambda_{\mathrm{QCD}}$, and $\Delta=4 \alpha_{s} N_{c} \ln 2 / \pi$. It is important that the third term in (2) is only non-zero at $\left|k_{T}\right| \ll \Lambda_{\mathrm{QCD}}(1 / x)^{\delta}$ with $\delta=\alpha_{s} N_{c}$. The details of the calculation and the relation between the TMD gluon density and the inclusive hadron spectra $\rho_{h}\left(y \simeq 0, p_{T}\right) \equiv$ $E d^{3} \sigma / d^{3} p$ are given in our previous papers[1,2]. These spectra are presented as a sum of two parts[7, 8]:

$$
\rho_{h}\left(y \simeq 0, p_{T}\right)=\rho_{q}\left(y \simeq 0, p_{T}\right)+\rho_{g}\left(y \simeq 0, p_{T}\right)
$$


where $\rho_{q}$ is the quark contribution calculated within the quark-gluon string model (QGSM) and $\rho_{g}$ is the gluon contribution, see [10], which can be calculated using the proposed TMD gluon density (1) and (2).
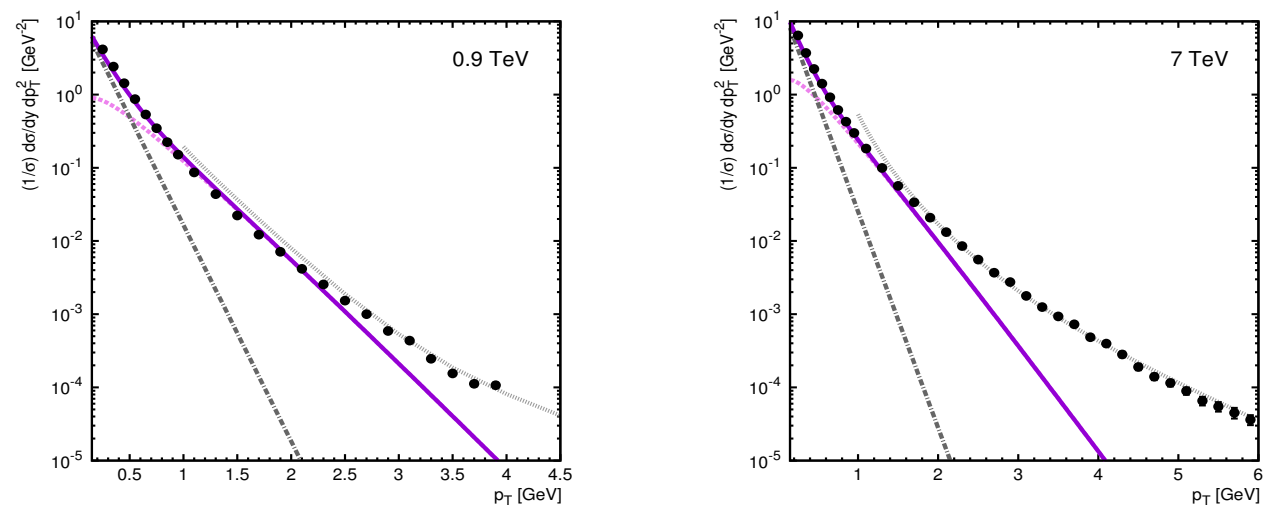

Figure 1: The inclusive cross sections of the hadron production in the $p p$ collisions at the LHC as a function of the transverse momentum. The dashed and dash-dotted curves correspond to the gluon and quark contributions, respectively. The solid curves represent their sum. The dotted curves correspond to the sum of the soft QCD and $\mathrm{pQCD}$ predictions as described in the text. The experimental data are from ATLAS and CMS[12].

\section{- CCFM-evolved TMD parton densities}

The average gluon transverse momentum $\left\langle\left|k_{T}\right|\right\rangle$, generated by the TMD gluon distribution defined above is $\left\langle\left|k_{T}\right|\right\rangle \sim 1.9 \mathrm{GeV}$ at $10^{-7}<x<1$, which is close to the non-perturbative QCD regime. Therefore, we can treat the proposed TMD gluon density as a starting one and apply the CCFM equation to extend it to the whole kinematical region. The CCFM evolution equation resumes large logarithms $\alpha_{s}^{n} \ln ^{n} 1 /(1-x)$ in addition to $\alpha_{s}^{n} \ln ^{n} 1 / x$ ones and introduces angular ordering of initial emissions to correctly treat gluon coherence effects. In the limit of asymptotic energies, it is almost equivalent to BFKL, but also similar to the DGLAP evolution for large $x$ and high $\mu^{2}$ [4]. In Fig. (2) he CCFM-evolved TMD gluon densities in the proton calculated as a function of the gluon transverse momentum $\mathbf{k}_{T}^{2}$ at $x=10^{-4}$ and two values of $\mu^{2}$ is presented. The details of the calculation are presented in [10].

The gluon density $f_{g}\left(x, k_{T}^{2}, \mu^{2}\right)$ obtained according to (1) and (2), labeled below as MoscowDubna 2015, or MD'2015, is shown in Fig. (02 as a function of $k_{T}^{2}$ for different values of $x$ and $\mu^{2}$. Additionally, we plot the TMD gluon distribution[11] (namely, the set A0) which is widely discussed in the literature and commonly used in the applications. One can observe some difference in the absolute normalization and shape between both TMD gluon distributions. Below we will consider the corresponding phenomenological consequences for several LHC processes.

\section{- Phenomenological applications}

As is well known, beauty quarks at the LHC energies are produced mainly via standard QCD gluon-gluon fusion subprocess; therefore, the corresponding total and differential cross sections are strongly sensitive to the gluon content of the proton. In the $k_{T}$-factorization approach this was demonstrated in our previous paper[13]. Below we probe the MD'2015 gluon density in the 

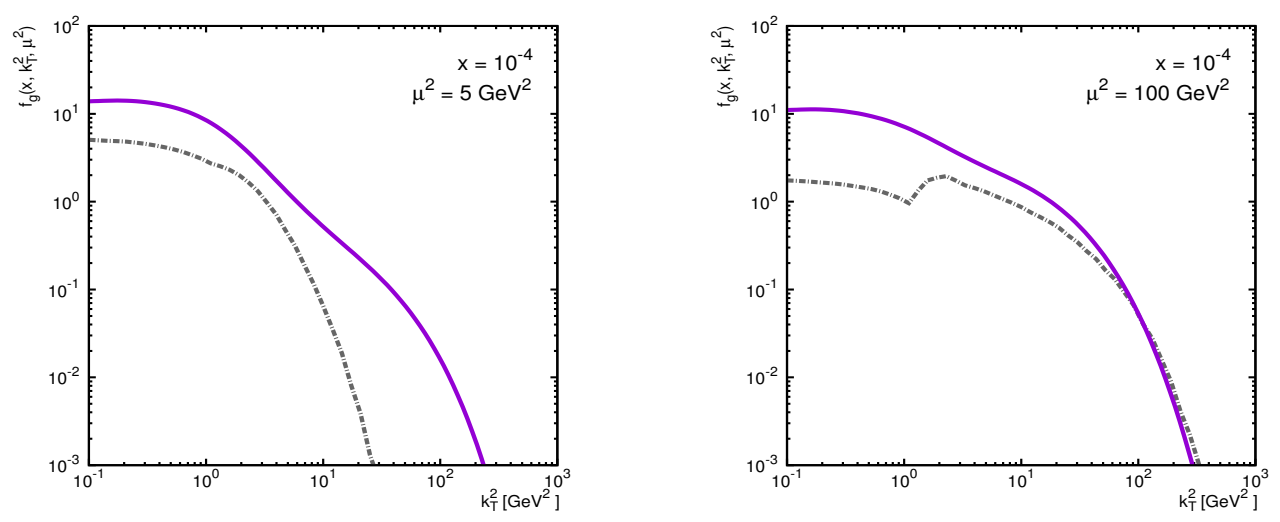

Figure 2: The CCFM-evolved TMD gluon densities in the proton calculated as a function of the gluon transverse momentum $\mathbf{k}_{T}^{2}$ at different $x$ and $\mu^{2}$. The solid and dashed curves correspond to the proposed MD'2015 and A0 gluon densities, respectively.

inclusive $b$-jet production at the LHC. The CMS Collaboration measured the $b$-jet cross sections in five $b$-jet rapidity regions, namely, $|y|<0.5,0.5<|y|<1,1<|y|<1.5,1.5<|y|<2$, and $2<|y|<2.2$ as a function of the jet transverse momentum at $\sqrt{s}=7 \mathrm{TeV}[14]$. The ATLAS Collaboration performed the measurements at central rapidities $|y|<2.1[15]$.

A part of our results are presented in Figs. (3) and (4), where we plot the calculated transverse momentum distributions of $b$-jets compared to the LHC data as well as the corresponding data/theory ratios. We obtained a good description of the data using the MD'2015 gluon distribution. The shape and absolute normalization of the measured $b$-jet cross sections are reproduced well. Moreover, the differential cross section as a function of the angular separation $\Delta \phi$ between two $b$-jets measured at $p_{T}>40 \mathrm{GeV}$ and $M>110 \mathrm{GeV}$, where $M$ is the invariant mass of the produced $b \bar{b}$ pair, is also well described. This observable is known to be very sensitive to the $k_{T}^{2}$-behavior of the TMD gluon distribution.

The predictions based on the A0 gluon density lie below the data at high $p_{T}>40-50 \mathrm{GeV}$. Once again, we conclude that the best description of the LHC data[14, 15] is achieved with the proposed MD'2015 gluon density. More detailed results of the suggested approach represented in our paper [10].

\section{- Conclusion}

We fitted the experimental data on the inclusive spectra of the charged particles produced in the central $p p$ collisions at the LHC to determine the TMD gluon density in a proton at the starting scale $\mu_{0}^{2} \sim 1 \mathrm{GeV}^{2}$. We demonstrated that the best description of these spectra can be achieved by matching the derived TMD gluon distribution with the exact solution of the Balitsky-FadinKuraev-Lipatov (BFKL) equation obtained at low $x$ and small gluon transverse momenta outside the saturation region. Moreover, we established that the parameters of this fit did not depend on the initial energy in a wide energy interval. The average gluon transverse momentum generated by this modified TMD gluon density is about $1.9 \mathrm{GeV}$ in a wide region of $x$ and is close to the non-perturbative QCD regime. Then, we extended the derived TMD gluon density to higher $\mu^{2}$ 

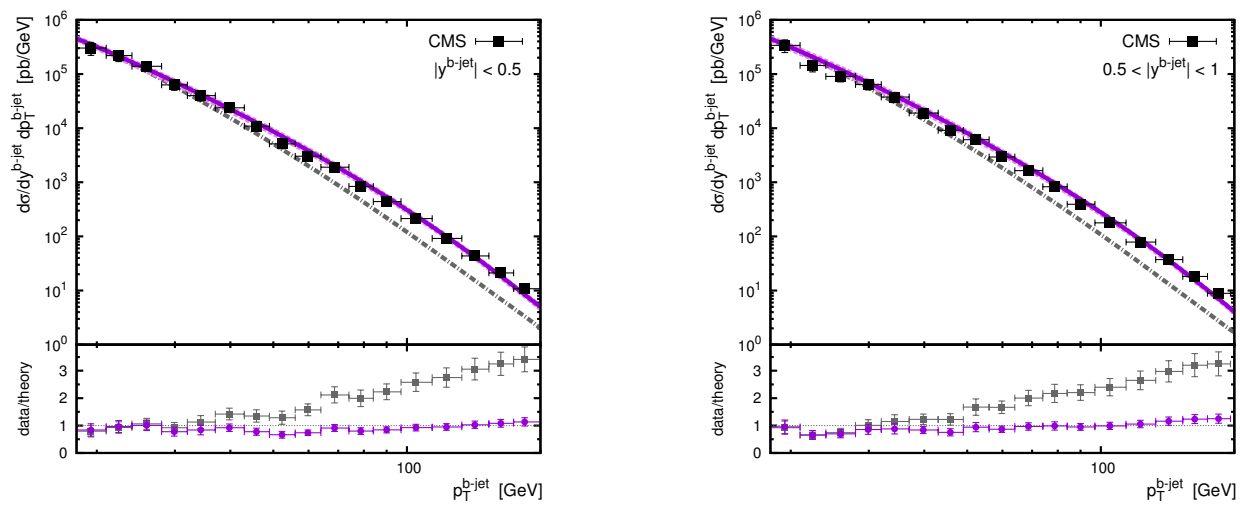

Figure 3: The double differential cross sections $d \sigma / d y d p_{T}$ of the inclusive $b$-jet production as a function of the leading jet transverse momentum in different $y$ regions. The used kinematical cuts are described in the text. The solid line corresponds to our calculation, the dash curve is the calculation using the set A0 of the unintegrated gluon density [11]. The experimental data are from CMS [14].
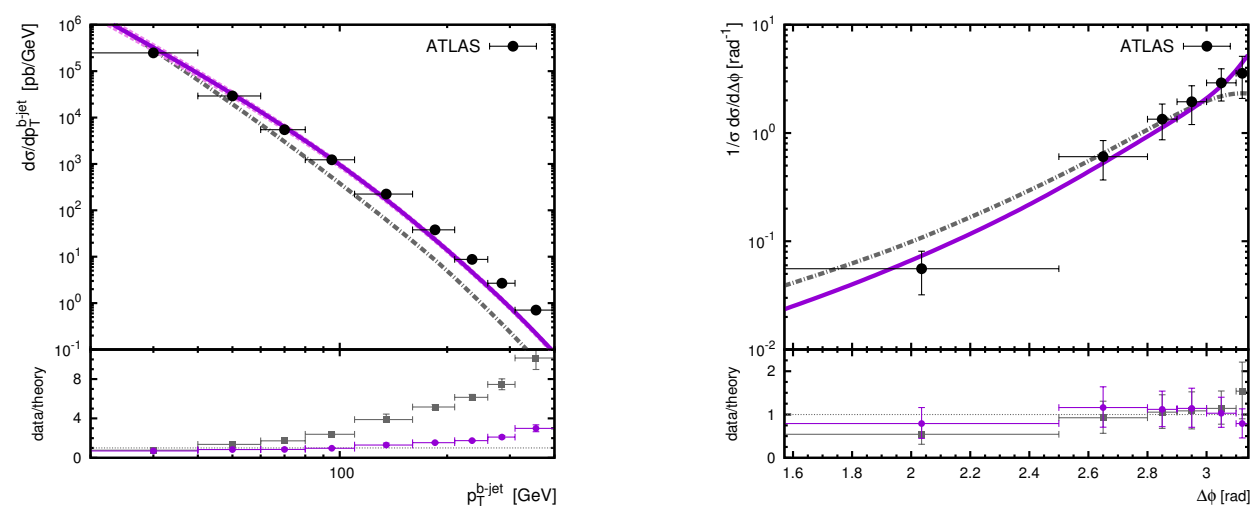

Figure 4: The distributions in the leading $b$-jet transverse momentum and azimuthal angle difference between the $b$-jets produced at the LHC. The used kinematical cuts are described in the text. Notation of all curves is the same as in Fig. (3). The experimental data are from ATLAS [15].

using numerical solution of the CCFM gluon evolution equation. Additionally, we supplied the calculated TMD gluon density with the TMD valence and sea quark distributions. The latter was evaluated in the approximation where the gluon-to-quark splitting occurred at the last evolution step using the TMD gluon-to-quark splitting function. This function contains all single logarithmic small- $x$ corrections in any order of perturbation theory.

Special attention was paid to the phenomenological applications of the proposed MD'2015 parton distributions to the hard processes. As for example, we presented here the results on the inclusive production of $b$-jets and correlation between two $b$-jets produced in $p p$ collision. In [10] 
our results on the inclusive production of $B^{+}$and $D^{*}$ mesons and the associated production of $W^{ \pm}$ or $Z / \gamma^{*}$ bosons and hadronic jets at the LHC energies are presented. We showed that the LHC data could be well described using the MD'2015.

\section{- Acknowledgements}

We thank H. Jung for his extreme help in the calculation of the CCFM evolution and very useful discussions and comments. We also thank L.N. Lipatov and B.I. Ermolaev for helpful discussion and M.A. Malyshev for careful reading of the manuscript. This research was supported by the FASI of Russian Federation (grant NS-3042.2014.2). A.V.L. and N.P.Z. are also grateful to the DESY Directorate for the support within the framework of the Moscow - DESY project on Monte-Carlo implementation for HERA - LHC.

\section{References}

[1] A.A. Grinyuk, A.V. Lipatov, G.I. Lykasov, N.P. Zotov, Phys. Rev. D 87, 074017 (2013).

[2] A.V. Lipatov, G.I. Lykasov, N.P. Zotov, Phys. Rev. D 89, 014001 (2014).

[3] K. Golec-Biernat, M. Wüsthoff, Phys. Rev. D 59, 014017 (1998);

K. Golec-Biernat, M. Wüsthoff, Phys. Rev. D 60, 114023 (1999).

[4] M. Ciafaloni, Nucl. Phys. B 296, 49 (1988);

S. Catani, F. Fiorani, G. Marchesini, Phys. Lett. B 234, 339 (1990);

S. Catani, F. Fiorani, G. Marchesini, Nucl. Phys. B 336, 18 (1990);

G. Marchesini, Nucl. Phys. B 445, 49 (1995).

[5] E.A. Kuraev, L.N. Lipatov, V.S. Fadin, Sov. Phys. JETP 44, 443 (1976);

E.A. Kuraev, L.N. Lipatov, V.S. Fadin, Sov. Phys. JETP 45, 199 (1977);

I.I. Balitsky, L.N. Lipatov, Sov. J. Nucl. Phys. 28, 822 (1978).

[6] V.N. Gribov and L.N. Lipatov, Sov.J. Nucl. Phys. 15, 438 (1972);

L.N. Lipatov, Sov. J. Nucl. Phys. 20, 94 (1975);

G. Altarelli, G. Parisi, Nucl. Phys. B 126, 298 (1977);

Yu.L. Dokshitzer, Sov. Phys. JETP 46, 641 (1977).

[7] D.A. Artemenkov, G.I. Lykasov, A.I. Malakhov, Int. J. Mod. Phys. A 30, 1550127 (2015); arXiv:1504.07841 [hep-ph].

[8] V.A. Bednyakov, A.A. Grinyuk, G.I. Lykasov, M. Poghosyan, Int. J. Mod. Phys. A 27, 1250042 (2012).

[9] Yu.V. Kovchegov, Phys. Rev. D61, 074018 (2000).

[10] A.A. Grinyuk, A.V. Lipatov, G.I. Lykasov, N.P. Zotov, Phys. Rev. D 93, 014035 (2016).

[11] H. Jung, arXiv:hep-ph/0411287.

[12] ATLAS Collaboration, New J. Phys. 13, 053033 (2011);

CMS Collaboration, Phys. Rev. Lett. 105, 022002 (2010).

[13] H. Jung, M. Kraemer, A.V. Lipatov, N.P. Zotov, Phys. Rev. D 85, 034035 (2012).

[14] CMS Collaboration, JHEP 1204, 084 (2012).

[15] ATLAS Collaboration, Eur. Phys. J. C 71, 1846 (2011). 